\title{
Magnetic Interactions in Tb and Tb-10\% Ho from Inelastic Neutron Scattering
}

Bjerrum Møller, Hans; Houmann, Jens Christian Gylden; Mackintosh, A.R.

Published in:

Journal of Applied Physics

Link to article, DOI:

$10.1063 / 1.2163623$

Publication date:

1968

Document Version

Publisher's PDF, also known as Version of record

Link back to DTU Orbit

Citation (APA):

Bjerrum Møller, H., Houmann, J. C. G., \& Mackintosh, A. R. (1968). Magnetic Interactions in Tb and Tb-10\% Ho from Inelastic Neutron Scattering. Journal of Applied Physics, 39(2), 807-815. https://doi.org/10.1063/1.2163623

\section{General rights}

Copyright and moral rights for the publications made accessible in the public portal are retained by the authors and/or other copyright owners and it is a condition of accessing publications that users recognise and abide by the legal requirements associated with these rights.

- Users may download and print one copy of any publication from the public portal for the purpose of private study or research.

- You may not further distribute the material or use it for any profit-making activity or commercial gain

- You may freely distribute the URL identifying the publication in the public portal 


\section{CONCLUSION}

The object of this paper has been to underline the pint (previously made by other authors but still often disegarded) that in the presence of orbital effects ineractions are not of the Heisenberg type. Their actual orm is restricted by symmetry and may be related by the above methods to microscopic models.

Detailed experimental investigation of such systems rould be welcome, either from pair spectra of ions in diute crystals from the excitation spectra in the ordered state. The low-lying excitations are spin waves and their detailed dispersion reflect the form and range of the exchange. There are also exciton bands corresponding to excitations of single-ions to higher atomic states which propagate across the crystal because of exchange. It is planned to study the theoretical form of those spectra using the more complicated spin Hamiltonians discussed above. For example $\mathrm{UO}_{2}{ }^{17}$ shows signs of anisotropy which presumably arises from the exchange.

${ }^{17}$ G. Dolling and R. Cowley, Phys. Rev. Letters 16, 683 (1966).

\title{
Magnetic Interactions in $\mathrm{Tb}$ and $\mathrm{Tb}-10 \%$ Ho from Inelastic Neutron Scattering
}

\author{
H. BJERRUM M $\phi$ LLEN \\ A.E.K. Research Establishmenl, Risó, Denmark \\ AND \\ J. C. Gylden Houmann and A. R. Mackintosh \\ Teclinical University, Lyngby, Denmark
}

\begin{abstract}
The magnon dispersion relations and lifetimes have been measured in $\mathrm{Tb}$ and a $\mathrm{Tb}-10 \%$ Ho alloy by inelastic neutron scattering, in regions of both ferromagnetic and spiral ordering. In the ferromagnetic phase, the magnon energy is generally finite at zero wavevector and rises quadratically at low $q$. The magnon energies scale approximately with the magnetization. In the spiral phase the magnon energy rises linearly from zero at low $q$. The Fourier-transformed exchange parameter $J(\mathrm{q})$ has pronounced peaks in the $c$ direction, which are ascribed to transitions between states close to the Fermi surface. These peaks are less pronounced in the ferromagnetic phase. The primary mechanism limiting the magnon lifetimes appears to be interaction with the conduction electrons. In the alloy, the lifetime for magnons propagating in the $c$ direction in the ferromagnetic phase falls abruptly at about $q=0.35 \AA^{-1}$, and this may be due to the exchange splitting of the conduction-electron energy bands. The dispersion curve for magnons propagating in the $a$ direction is considerably perturbed around $4 \mathrm{meV}$, and this is probably due to resonant scattering on the Ho impurities. A strong coupling occurs over the same energy range in the alloy between magnons and transverse phonons propagating in the $c$ direction. This effect is considerably smaller in pure Tb.
\end{abstract}

\section{INTRODUCTION}

The experiments described in this paper were undersen with the aim of improving the understanding of magnetic interactions in rare earth metals, by sedying the dynamics of the magnetic systems in ter ordered phases. A considerable amount of informawhout these interactions has been obtained from her experiments, especially from measurements of static magnetic susceptibility ${ }^{1}$ and neutron diffracSon studies ${ }^{2}$ of the frequently complex magnetic structs On the other hand, the dynamical properties of * magnetic systems are a much richer source of intatic on the magnetic interactions than are the thic properties alone, just as the study of lattice dythrics provides detailed information on interatomic ID. E, Hegland

44. 158 (1963) t. C. C. Koeh \begin{tabular}{l} 
5. Tol. Phys. 34, H. R. Child, E. O. Wollan, and J. W. Cable, \\
\hline
\end{tabular} forces which a knowledge of the lattice structure alone does not.

Inelastic neutron scattering has proved to be an ideal technique for such a study, since the neutron interacts strongly with uncompensated magnetic moments, undergoing changes in energy and momentum which can readily be measured, while the scattering cross section is determined essentially by the timedependent pair correlation function for these moments. ${ }^{3}$ Many of the rare earth metals have undesirably high capture cross sections for thermal neutrons, but $\mathrm{Tb}$ is relatively favorable in this respect and has the additional advantage of a large magnetic moment, so that the magnetic scattering cross section is large. The results presented in this paper are therefore exclusively on $\mathrm{Tb}$ and a dilute alloy with $10 \% \mathrm{Ho}$, although magnetic excitations have recently been observed in $\mathrm{Er}^{4}$

\footnotetext{
${ }^{3}$ L. Van Hove, Phys. Rev. 951374 (1954).

${ }_{4}^{4}$ A. D. B. Woods, T. M. Holden, and B. M. Powell, Phys. Rev. Letters 19, 908 (1967).
} 
and $\mathrm{Ho},{ }^{5}$ which also have reasonable neutron properties.

In the next section we describe briefly the experimental method, with especial attention to those features of the technique which allow an accurate determination of the energies and lifetimes of the magnons. The experimental results, some of which have been briefly reported previously, ${ }^{6,7}$ are then presented. The interpretation of these data is discussed in the following section, with special reference to the relation between the conduction-electron band structure and the indirect exchange interaction between the magnetic ions. The interaction between the magnons and electrons, phonons and impurities is also considered. Finally a summary is given of the information which these experiments have provided, and plans and suggestions for future work in this field are briefly discussed.

\section{EXPERIMENTAL TECHNIQUE}

The magnon dispersion relations and lifetimes were measured by inelastic neutron scattering, using the Riso triple axis spectrometer. The details of the experimental technique have been presented elsewhere. ${ }^{8}$

The scattering cross sections for one-magnon creation have the form

$$
d^{2} \sigma / d \Omega d E \sim \sum_{\tau}\left(1+e_{m}^{2}\right) \delta(\boldsymbol{v}-\tau-\mathbf{q}) \delta[E-\epsilon(\mathbf{q})]
$$

for the ferromagnetic phase, and

$$
\begin{aligned}
d^{2} \sigma / d \Omega d E \sim \sum_{\tau}\left\{1+e_{Q}{ }^{2}\right) f^{-1}(\mathbf{q})[\delta(\boldsymbol{\kappa}-\tau-\mathbf{q}-\mathbf{Q}) & \\
+\delta(\boldsymbol{\kappa}-\boldsymbol{\tau}-\mathbf{q}+\mathrm{Q})]+4\left(1-e_{Q}{ }^{2}\right) & f(\mathbf{q}) \delta(\boldsymbol{\kappa}-\boldsymbol{\tau}-\mathbf{q})\} \\
& \times \mathbf{\delta}[E-\boldsymbol{\epsilon}(\mathbf{q})]
\end{aligned}
$$

for a spiral structure with wavevector Q. Here $\tau$ is a reciprocal lattice vector, $\epsilon(\mathbf{q})$ is the energy of a magnon of wavevector $\mathbf{q}$, and $f(\mathbf{q})$ is a slowly varying function of q. $\boldsymbol{k}=\mathrm{k}_{1}-\mathrm{k}_{2}$ is the neutron scattering vector, where $\mathbf{k}_{1}$ and $\mathbf{k}_{2}$ are the wavevectors of the incident and scattered neutrons. $e_{m}$ and $e_{Q}$ are the components of the unit scattering vector in the direction of the magnetization and spiral wavevector respectively.

The natural variables of a neutron scattering experiment are the energy transfer $E=\hbar^{2} / 2 m_{0}\left(k_{1}{ }^{2}-k_{2}{ }^{2}\right)$ and the vector $\mathrm{q}=\boldsymbol{\kappa}-\tau$. The experimental method therefore consists of seeking peaks in the scattered neutron intensity by varying $\mathrm{q}$ (constant $E$ scans) or $E$ (constant q scans). As may be seen from the cross-section formulas, the positions of such neutron groups deter-

${ }^{5}$ H. B. Møller, J. C. G. Houmann, and A. R. Mackintosh (to be published)

${ }^{6}$ H. B. Møller and J. C. G. Houmann, Phys. Rev. Letters 16, 737 (1966).

${ }^{7} \mathrm{H}$. Bjerrum Mфller, J. C. G. Houmann, and A. R. Mackintosh, Phys. Rev. Letters 19, 312 (1967).

${ }^{8} \mathrm{H}$. B. M $\varnothing$ ller, thesis submitted to the University of Copenhagen (1967). mine the dispersion relation $\epsilon(\mathbf{q})$, and the width of the neutron groups gives the magnon lifetimes, provides that the instrumental resolution can be extracted Neutron groups are also produced by phonon scat tering, but the cross sections in the crystals which we studied are much smaller, except at very low energ transfers. Any disturbance from phonon scattering can generally be eliminated by an appropriate choice of the relative directions of the polarization vectors and $\boldsymbol{x}$.

For the spiral structure, three neutron groups sepa rated by an interval $Q$ are generally observed in a constant $E$ scan in the $c$ direction. However, if a scan is made for which $\boldsymbol{\kappa}$ is along $\mathrm{Q}$, the center peak is eliminated, so that resolution of the remaining two is easier. Figure 1 shows the results of such a constant $E$ scan for Tb-10\% Ho in the spiral phase.

Because of the finite angular collimations of the neutron beam and the mosaic spread of the monochromator, sample and analyzer crystals, the variables $\mathrm{q}$ and $E$ are not sharply defined but have a distribution about their average values $\mathrm{q}_{0}$ and $E_{0}$. The probability of obtaining the values $\mathrm{q}$ and $E$ with the spectrometer set at $\mathrm{q}_{0}, E_{0}$ is defined as the resolution function $R(\mathbf{q}-\mathrm{q}$ $\left.E-E_{0}\right)$. If the cross section is $\sigma(\mathrm{q}, E)$, we then measure the intensity

$$
I\left(\mathbf{q}_{0}, E_{0}\right)=\iint \sigma(\mathbf{q}, E) R\left(\mathbf{q}-\mathbf{q}_{0}, E-E_{0}\right) d \mathbf{q} d E .
$$

For a Bragg cross section, proportional to $\delta(\mathbf{q}) \delta(E)$, we find

$$
I^{B}\left(\mathrm{q}_{0}, E_{0}\right) \sim R\left(-\mathrm{q}_{0},-E_{0}\right) .
$$

The resolution function can therefore be measured for $\mathrm{q}_{0}=0$ and $E_{0}=0$ by mapping the Bragg reflected intensity as a function of the spectrometer setting. Since the resolution function only depends on the distributions of $\mathbf{k}_{1}$ and $\mathbf{k}_{2}$ about their averages, it is a slowly

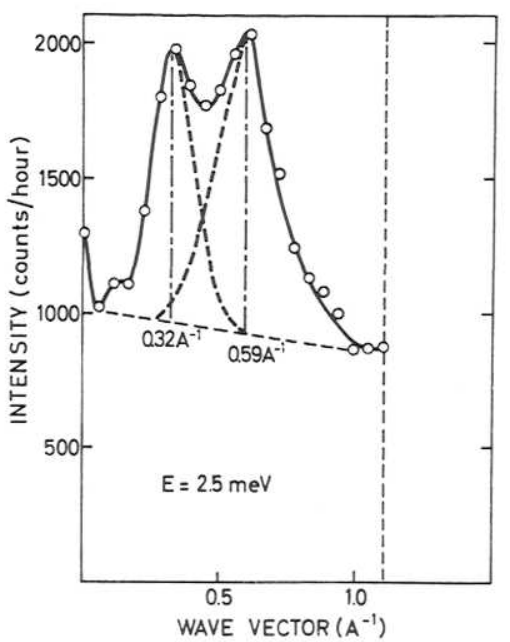

Fig. 1. Neutron groups from a cos: stant- $E$ scan in the spiral phase of Tb$10 \%$ Ho at $200 \%$

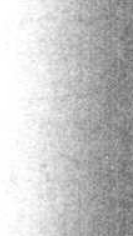


Fic. 2. Experimental magnon disperin relations in $\mathrm{Tb}$ along symmetry lines in the Brillouin zone at $90^{\circ} \mathrm{K}$.
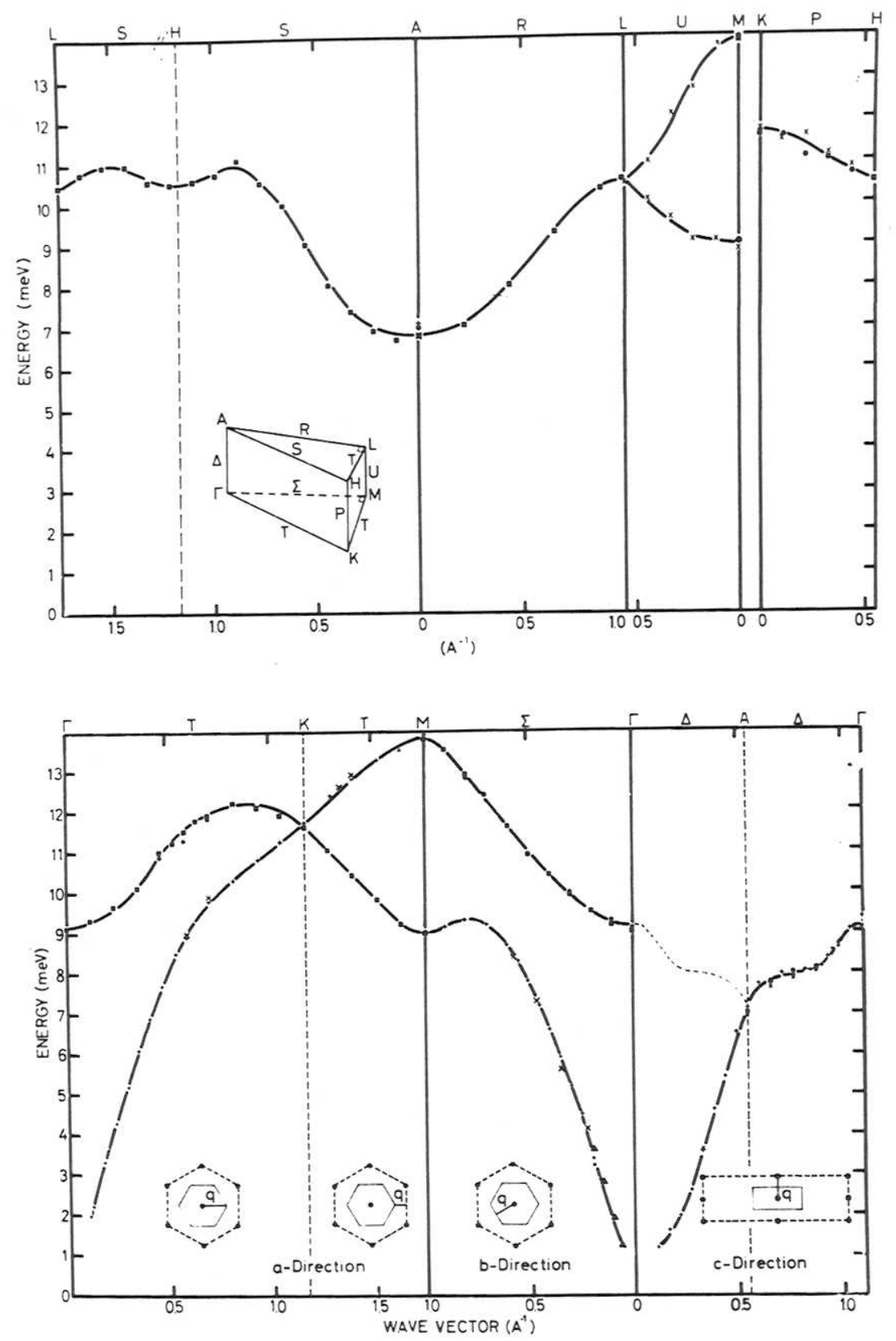

Prying function of $\mathrm{q}_{0}$ and $E_{0}$, so that its variation with find $E_{0}$ can be neglected when $\left|\mathrm{q}_{0}\right|$ is small compared * $\mathbf{k}_{2}$, and $E_{0}$ is small compared with the energy too requirered neutrons. In this experiment, these fopoquirements were always fulfilled, so that the 74 s.ion function measured on a Bragg reflection onsured to be applicable to the inelastic-scattering Thements.

There is a strong correlation between $\mathrm{q}$ and $E$, thing $\mathrm{p}$ when $\mathrm{q}$ is perpendicular to $\boldsymbol{k}$ and in the scatGione. This leads to focusing effects. A dispersion 1.6, 6 tobained with $\boldsymbol{x}$ perpendicular to $\mathrm{q}$ is shown in together with the half-value contour line of the resolution function. There is a strong correlation between $E$ and this component of q, whereas no correlation between $E$ and the other two components of q was found. In a constant q scan this resolution function is moved vertically and when it crosses the dispersion relation, a neutron group is observed, whose width depends on the size and orientation relative to the dispersion curve of the resolution function, and the natural width of the magnon. A knowledge of the resolution function therefore allows a determination of the resolution width for any scan. This is an important consideration when choosing scans, and in determining the natural width of the neutron groups. 


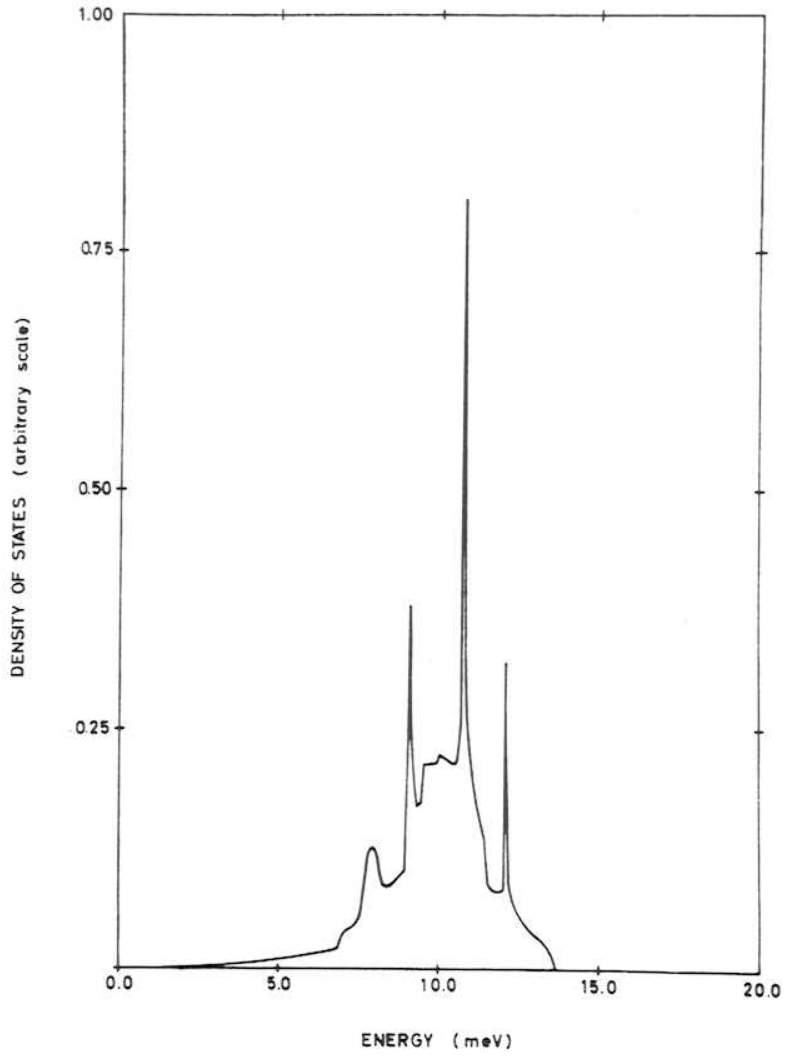

FIG. 3. Smoothed computer plot of the density of states for magnons in $\mathrm{Tb}$ at $90^{\circ} \mathrm{K}$. The curve is normalized so that the total number of states is unity.

\section{EXPERIMENTAL RESULTS}

The magnon dispersion relations for $\mathrm{Tb}$ at $90^{\circ} \mathrm{K}$ along all the symmetry lines in the Brillouin zone are shown in Fig. 2. The uncertainty in the measured magnon energies is estimated to be $\pm 0.1 \mathrm{meV}$. The magnon energy is finite at $\Gamma$ and rises quadratically for small q. The degeneracy over the hexagonal face of the zone

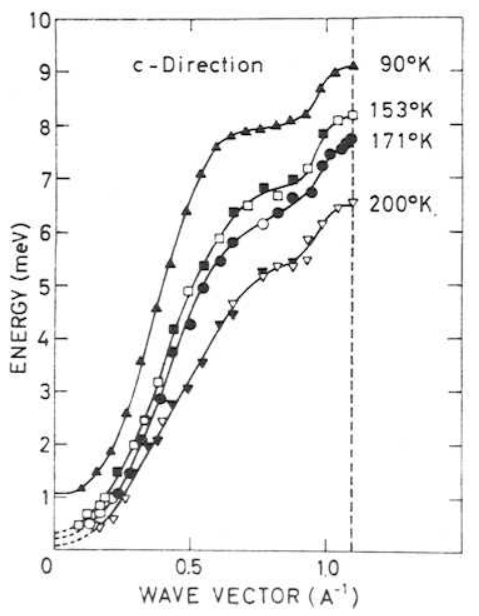

Fig. 4. Temperature dependence of magnon energies in the $c$ direction in the ferromagnetic phase of Tb. and along the line $K H$ are consistent with the symmet of the spin space group.9 The kink in the dispers curve along $\Gamma A$ is a noteworthy feature of thesersion urements. An analytical interpolation scheme meas been used to obtain magnon energies throughout zone from the results of Fig. 2, and this method the found to give results consistent, within the mental accuracy, with further measurements ox metry planes and at general points within the zone. Ty resulting magnon density of states is shown in $\mathrm{F}$ The energy gap at $\Gamma$ and Van Hove singularitio. evident in this plot.
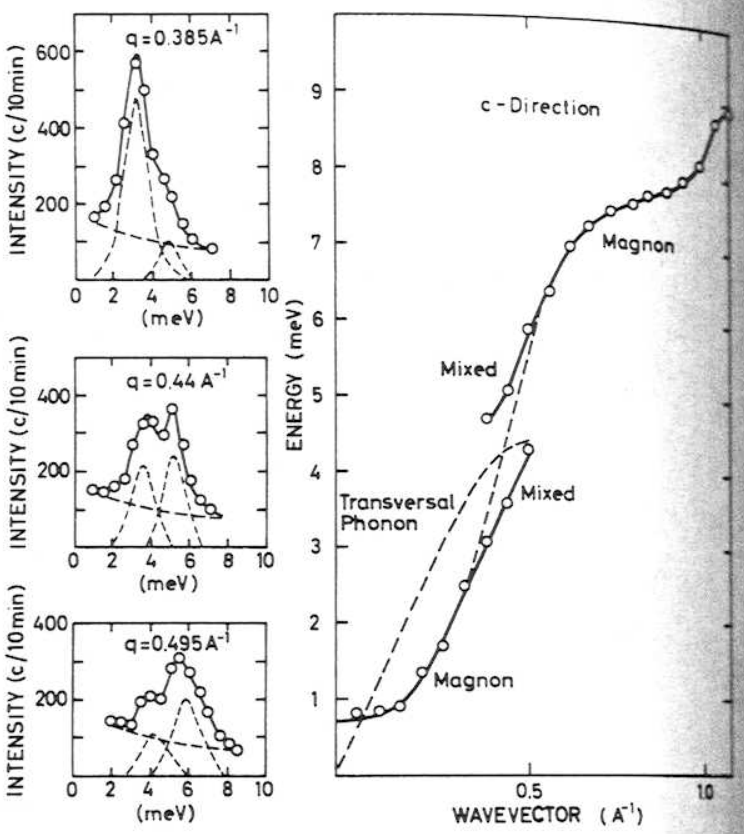

FIg. 5. Neutron groups and excitation energies in the direction of $\mathrm{Tb}-10 \% \mathrm{Ho}$ at $110^{\circ} \mathrm{K}$. The scan used for thes measurements was such that transverse phonons were aot observed.

The magnon energies along $\Gamma A$ have also been studied as a function of temperature in the ferromagnetic phase, and the dispersion curves in the double zone rep. resentation are shown in Fig. 4. The magnon energies decrease with temperature and the energy gap at I falls rapidly to a low value.

Similar measurements in the ferromagnetic phase of a Tb-10\% Ho alloy are shown in Fig. 5. The general form of the dispersion relation is similar to that in $\mathrm{Tb}$, but it is rather flatter at low $\mathrm{q}$, and where it crosses the dispersion curve for the transverse phonons, there is a strong magnon-phonon coupling which causes a mixing of the modes and a splitting into two branches. The magnon-phonon interaction was also observed in pure $\mathrm{Tb}$, by carefully studying the region of crossing of the

${ }^{9}$ W. Brinkman, J. Appl. Phys. 38, 939 (1967).

${ }^{10} \mathrm{~J}$. C. G. Houmann (to be published). 

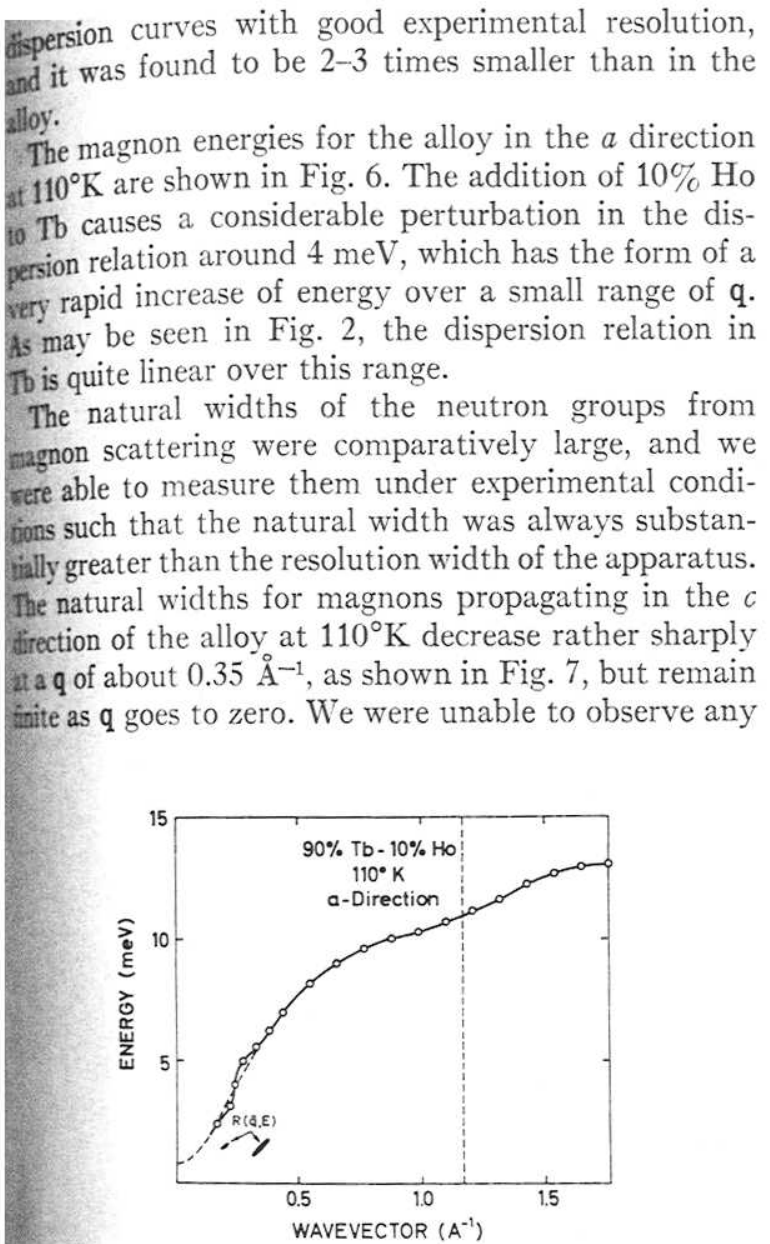

Fit. 6. Magnon dispersion relation in the $a$ direction for $10 \%$ Ho at $110^{\circ} \mathrm{K}$. The experimental resolution functions for he tro different resolutions used are also shown.

Cange in the lifetime of the magnon of highest $\mathrm{q}$ as the emperature was increased to $190^{\circ} \mathrm{K}$. The magnon nidhs in pure $\mathrm{Tb}$ were also measured at small and arge $\mathrm{q}$ in the $c$ direction at $90^{\circ} \mathrm{K}$, and were found to be, whin the experimental error, the same as those in the toy.

Because of the small temperature range of spiral ecter in $\mathrm{Tb}$, it was not possible to measure the magnon ispersion relation satisfactorily in the spiral phase. the addition of $10 \%$ Ho increases the tendency towards that thation of a periodic magnetic structure however, that the spiral phase is stable from $195^{\circ}$ to $221^{\circ} \mathrm{K}$. We to therefore able to measure the dispersion relation magnons in the spiral phase of the alloy, and the ints for the $c$ direction are shown in Fig. 8. The toon energy rises linearly from zero at $\Gamma$ and is Wole than that in the ferromagnetic phase over the scoult range. The measurements at higher $q$ were toxps.

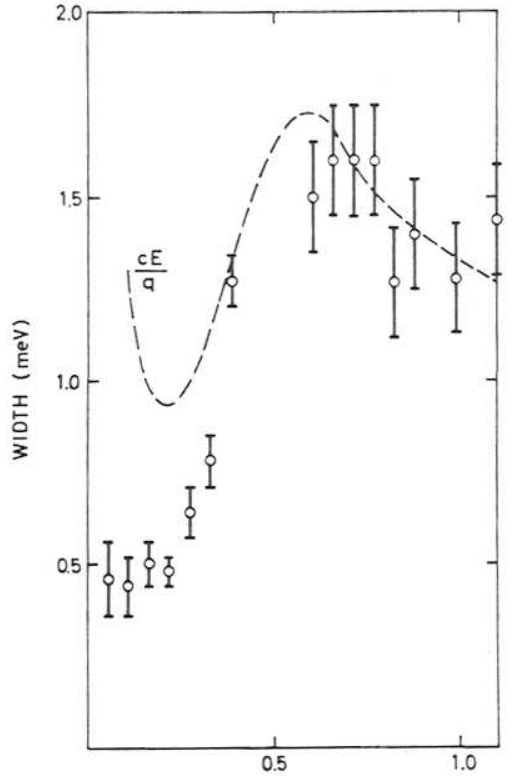

WAVEVECTOR $\left(A^{-1}\right)$

FIG. 7. Natural width of magnons propagating in the $c$ direction of $\mathrm{Tb}-10 \% \mathrm{Ho}$ at $110^{\circ} \mathrm{K}$. The experimental resolution, which is always smaller than the natural width, has been extracted from the measurements.

\section{DISCUSSION}

In order to interpret our results, we use the simplest Hamiltonian which describes the anisotropic magnetic structures of $\mathrm{Tb}$ and is consistent with our measured dispersion relations. We therefore write

$$
\begin{aligned}
H=-\sum_{i<j} J\left(\mathbf{R}_{i}-\mathbf{R}_{j}\right) \mathbf{S}_{i} \cdot \mathbf{S}_{j}+\sum_{j}\left\{B S_{z j}{ }^{2}\right. \\
\left.+\frac{1}{2} G\left[\left(S_{x j}+i S_{y j}\right)^{6}+\left(S_{x j}-i S_{y j}\right)^{6}\right]\right\},
\end{aligned}
$$

where $J\left(\mathbf{R}_{i}-\mathbf{R}_{j}\right)$ represents the indirect exchange interaction ${ }^{11}$ between ions at $\mathbf{R}_{i}$ and $\mathbf{R}_{j}$, and $B$ and $G$

FIG. 8. Magnon dispersion relations in $\mathrm{Tb}-10 \%$ Ho in the ferromagnetic and spiral phases. The full line for the ferromagnetic phase is derived principally from the experimental points of Fig. 5, while the form of the magnon dis. persion curve in the region of the magnon-phonon in- $z_{w}$ teraction is determined from measurements at higher temperatures. The full line for the spiral phase is a weighted least-squares fit of the experimental points to Eq. (16).

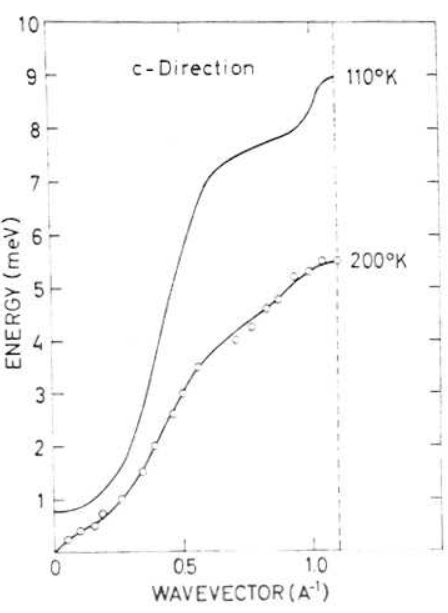

${ }^{11}$ M. A. Ruderman and C. Kittel. Phvs. Rev. 96, 99 (1954). 


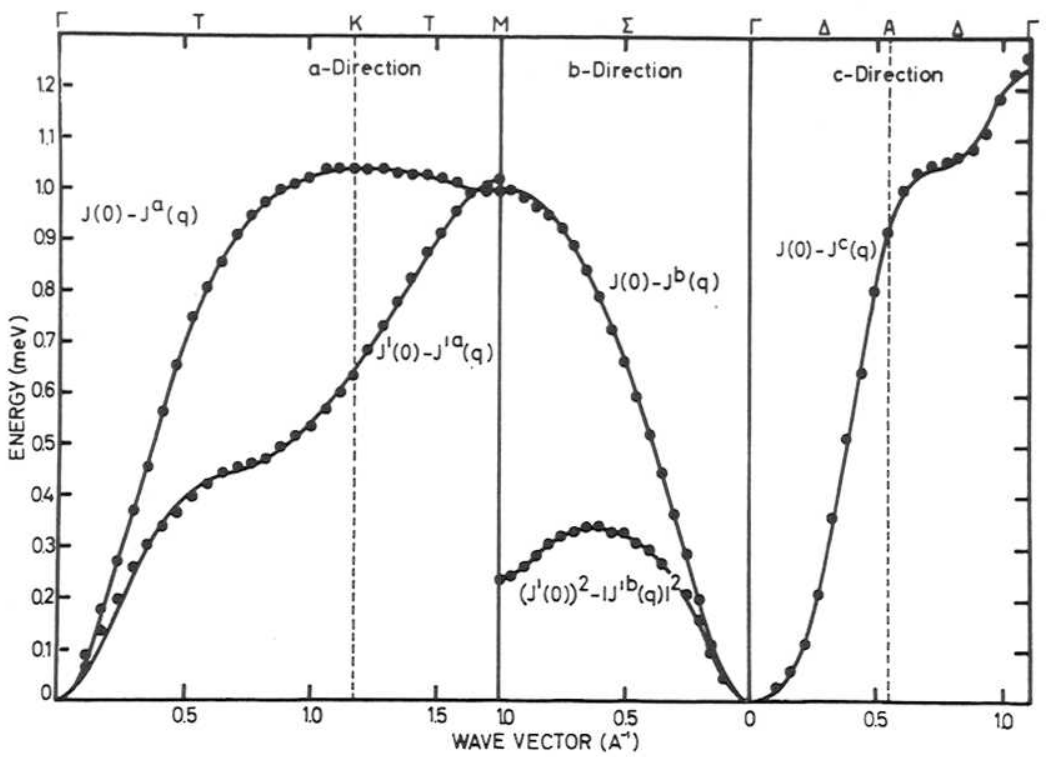

FIG. 9. Fourier-transformed exchange parameters for $\mathrm{Tb}$ at $90^{\circ} \mathrm{K}$.

are single-ion anisotropy parameters describing the twofold and sixfold anisotropy, respectively. We have used $\mathbf{S}$ to denote the total angular momentum operator for the ions. We shall analyze the magnon dispersion relations in terms of the Fourier transforms of the indirect exchange parameters, defined by

$$
\begin{gathered}
J(\mathbf{q})=\sum_{\mathbf{R}_{j}} J\left(\mathbf{R}_{j}\right) \exp \left(i \mathbf{q} \cdot \mathbf{R}_{j}\right) \\
J^{\prime}(\mathbf{q})=\sum_{\mathbf{R}_{j^{\prime}}} J\left(\mathbf{R}_{j}{ }^{\prime}\right) \exp \left(i \mathbf{q} \cdot \mathbf{R}_{j}{ }^{\prime}\right),
\end{gathered}
$$

where, in the hcp structure, unprimed vectors lie between ions in the same sublattice and primed vectors between ions in different sublattices. An approximate diagonalization of the Hamiltonian in the ferromagnetic phase $\mathrm{e}^{12}$ then gives the magnon energies

$\epsilon_{j}(\mathbf{q})=S\left\{f_{j}^{2}+2\left(B+21 G S^{4}\right) f_{j}+72 G S^{4}\left(B+3 G S^{4}\right)\right\}^{1 / 2}$,

where

$$
f_{j}(\mathbf{q})=J(0)-J(\mathbf{q})+J^{\prime}(0)+(-1)^{j}\left|J^{\prime}(\mathbf{q})\right|
$$

and the subscripts $j=1$ and 2 refer, respectively, to the acoustic and optical magnon branches. For the $a$ and $b$ directions, $J(\mathrm{q})$ and $J^{\prime}(\mathrm{q})$ can be written in terms of interplanar exchange parameters $J_{m}$ as

$$
\begin{array}{r}
J(0)-J^{a}(q)=2 \sum_{m=1}^{\infty} J_{m}{ }^{a}\left(1-\cos \frac{1}{2} m a q\right), \\
(-2 \pi / a<q<2 \pi / a) \\
J^{\prime}(0)-J^{\prime a}(q)=2 \sum_{m=1}^{\infty} J_{m}{ }^{\prime a}\left(1-\cos \frac{1}{2} m a q\right), \\
(-2 \pi / a<q<2 \pi / a)
\end{array}
$$

${ }^{12}$ K. Niira, Phys. Rev. 117, 129 (1960). and

$$
\begin{aligned}
& J(0)-J^{b}(q)=2 \sum_{m=1}^{\infty} J_{m}{ }^{b}[1-\cos (\sqrt{3} / 2) m a q] \text {, } \\
& (-2 \pi / \sqrt{3} a<q<2 \pi / \sqrt{3} a) \\
& \left|J^{\prime}(0)\right|^{2}-\left|J^{\prime b}(q)\right|^{2} \\
& =\sum_{m=-\infty}^{\infty} \sum_{p=1}^{\infty} J_{m}{ }^{\prime b} J_{m-p}{ }^{\prime b}[1-\cos (\sqrt{3} / 2) m a q], \\
& (-2 \pi / \sqrt{3} a<q<2 \pi / \sqrt{3} a) \text {. (11 }
\end{aligned}
$$

For the $c$ direction, the ions, in the two sublattices for alternate equally separated planes and these expression can be simplified. The dispersion relation can be con sidered as a single acoustical branch running to th (001) reciprocal lattice point and if, for this direction we take the sum in Eq. (6) over all ionic sites, we har

$$
\begin{aligned}
\epsilon(\mathbf{q})= & S\left[(J(0)-J(\mathbf{q}))^{2}+2\left(B+21 G S^{4}\right)\right. \\
& \left.\times(J(0)-J(\mathbf{q}))+72 G S^{4}\left(B+3 G S^{4}\right)\right]^{1 / 2} .
\end{aligned}
$$

We now require only a single set of interplanar ex change constants, such that

$$
\begin{aligned}
& J(0)-J^{c}(\mathbf{q})=2 \sum_{m=1}^{\infty} J_{m}^{c}\left(1-\cos \frac{1}{2} m c q\right) \\
&(-2 \pi / c<q<2 \pi / c)
\end{aligned}
$$

where odd values of $m$ represent the exchange betwee planes in different sublattices.

The Fourier-transformed exchange parameters $90^{\circ} \mathrm{K}$, deduced from the average magnon energies of tained in different scans, are shown in Fig. 9. A leas squares fit of Eqs. (10), (11), and (13) to the data also shown in Fig. 9, and the interplanar exchane parameters thus obtained are given in Table $\mathbf{I}$. To 
mer of Fourier components used in the analysis s the minimum required to fit the data within the experimental accuracy.

The anisotropy constants used in this analysis were btained from the neutron scattering data. $B$ was determined from the variation with $\mathrm{q}$ of the neutron roup intensity in the $c$ direction. According to Lindard $e t a l{ }^{13}$ the twofold anisotropy introduces an extra ctor $\left(\left\{1+[B S / \epsilon(\mathbf{q})]^{2}\right\}^{1 / 2}+B S / \epsilon(\mathbf{q})\right)$ into the magon scattering cross section, and from our intensities we deatuce the value $B=0.25 \pm 0.05 \mathrm{meV} /$ ion at $110^{\circ} \mathrm{K}$. From Eq. (12) the energy gap at $\Gamma$ is given by

$$
S \Delta=S\left[72 G S^{4}\left(B+3 G S^{4}\right)\right]^{1 / 2},
$$

and from the measured energy gap at $110^{\circ} \mathrm{K}$ and the ralue of $B$ quoted above, we find $G S^{4}=(8.7 \pm 3.5) \times 10^{-4}$ $\mathrm{meV} /$ ion. Because of the greater accuracy of the data, the measurements on the alloy were used in this analyis, but it may be assumed that the addition of $10 \% \mathrm{Ho}$ does not drastically affect the anisotropy constants.

If we use the classical relations ${ }^{12}$ between the macroscopic and microscopic anisotropy constants

$$
K_{2} \simeq \frac{2}{3} B S^{2}, \quad K_{6}{ }^{6} \simeq G S^{6},
$$

re find quite good agreement between our value for $B$ and the value of $0.33 \mathrm{meV} /$ ion deduced from torque beasurements by Rhyne and Clark, ${ }^{14}$ but our value for $G$ is about six times smaller than they obtained from tagnetostriction measurements. A quantum mechanial treatment of the macroscopic anisotropy parame$\mathrm{krs}^{\text {,t }}$ taking into account the dependence of the zeropint magnon energy on magnetization, substantially rodifies both Eqs. (14) and (15) however, and this ay account for the discrepancy. In interpreting our scult, we have therefore used the anisotropy constants

TasLs I. Interplanar exchange parameters. All are given in $\Rightarrow v$ except the $J^{\prime}$ for the $b$ direction, which are in $\mathrm{meV}^{2}$. Actracy $\pm 0.005 \mathrm{meV}$.

$a$ direction $\quad b$ direction $\quad c$ direction

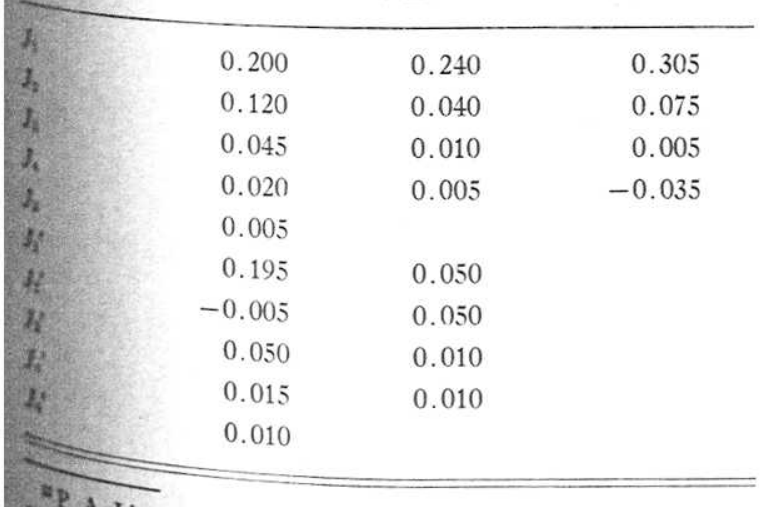

A. Aindgård, A. Kowalska, and P. Laut, J. Phys. Chem. ]. 28,1357 (1967).

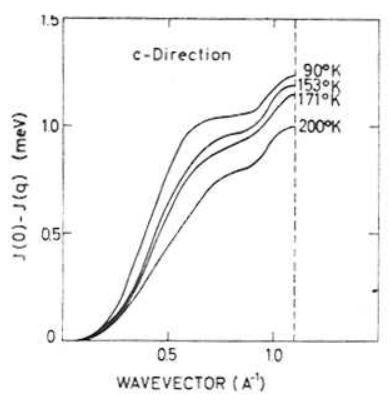

(a)

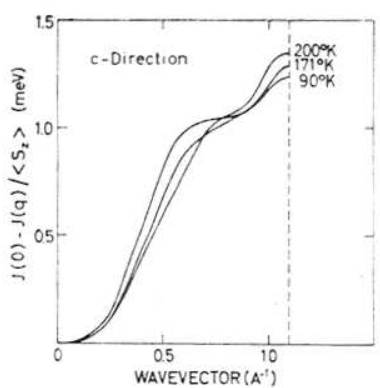

(b)
FIg. 10. (a) Temperature dependence of the Fourier-transformed exchange parameters in the $c$ direction in the ferromagnetic phase of Tb. (b) $J(0)-J(\mathrm{q})$ divided by the ordered moment in the ferromagnetic phase of $\mathrm{Tb}$.

derived from the neutron measurements at $110^{\circ} \mathrm{K}$, but scaled with temperature according to the results of Rhyne and Clark. ${ }^{14}$ Fortunately, the values of $J(\mathbf{q})$ deduced from the magnon dispersion curves are rather insensitive to variations in the anisotropy constants. $J(0)-J(\mathrm{q})$ in the $c$ direction is shown as a function of temperature in the ferromagnetic phase of $\mathrm{Tb}$ in Fig. 10. According to the theory of Tyablikov, ${ }^{16}$ which should be most valid at high temperatul s;, the exchange coupling between the ions should scale as the ordered moment, and we have therefore also plotted in Fig. 10 the function $[J(0)-J(\mathbf{q})] /\left\langle S_{z}\right\rangle$ for different temperatures. There is an approximate proportionality between the exchange constants and the moment, but the detailed form of $J(\mathrm{q})$ changes with temperature, and this reflects the change in energy-band structure with ordering, as well as the limitations of the theory.

A necessary condition for the stability of the spiral structure is a maximum in $J(\mathrm{q})$ at some nonzero $\mathrm{q}$, which is the wavevector $\mathbf{Q}$ of the spiral. The stronger tendency towards spiral ordering in the alloy, relative to that in pure $\mathrm{Tb}$, is reflected in the flattening of the dispersion curve, and hence of $J(\mathrm{q})$ at small q. The magnon dispersion relation in the $c$ direction in the spiral phase is ${ }^{17}$

$$
\begin{aligned}
\epsilon(\mathbf{q})=S\left(\left[J(\mathbf{Q})-\frac{1}{2} J\right.\right. & \left.(\mathbf{Q}+\mathbf{q})-\frac{1}{2} J(\mathbf{Q}-\mathbf{q})\right] \\
& \times[J(\mathbf{Q})-J(\mathbf{q})+2 B])^{1 / 2} .
\end{aligned}
$$

From the measured magnon energies, $J(\mathrm{q})$ can therefore be deduced by an iterative procedure, since $Q$ is known from neutron-diffraction measurements, and the results which we thereby obtain are shown in Fig. 11 . The incipient maxima in $J(\mathbf{q})$ in the ferromagnetic phase are developed in the spiral phase and occur around $\mathrm{Q}$ and $\tau-\mathrm{Q}$, where $\tau$ is the (001) reciprocal lattice vector. The condition for stability of the spiral structure is therefore satisfied.

${ }^{16}$ S. V. Tyablikov, Ukr. Math. Zh. 11, 287 (1959). See also H. B. Callen, Phys. Rev. 130, 890 (1963).

${ }^{17} \mathrm{~K}$. Yosida and H. Miwa, J. Appl. Phys. 32, 85 (1961). 


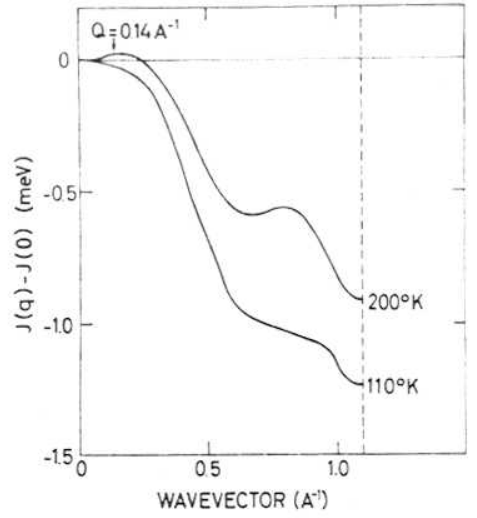

Fig. 11. $J(q)-J(0)$ for $\mathrm{Tb}-10 \%$ Ho deduced from the dispersion curves of Fig. 8 us ing Eqs. (12) and (16). The dashed line is drawn at the position of the 001) reciprocal lattice point.

$J$ (q) is directly related to the conduction-electron energy band structure and, in the periodic zone scheme, is given by an expression of the form ${ }^{18}$

$$
J(\mathbf{q})=\sum_{n, n^{\prime}, \mathbf{k}} \frac{I_{n n^{\prime}}(\mathbf{k}, \mathbf{k}+\mathbf{q})\left[f\left(\epsilon_{n}(\mathbf{k})-f\left(\epsilon_{n^{\prime}}(\mathbf{k}+\mathbf{q})\right)\right]\right.}{\epsilon_{n^{\prime}}(\mathbf{k}+\mathbf{q})-\epsilon_{n}(\mathbf{k})},
$$

where $\epsilon_{n}(\mathbf{k})$ is the energy of a Bloch state of wavevector $\mathbf{k}$ in band $n, I_{n n^{\prime}}(\mathbf{k}, \mathbf{k}+\mathbf{q})$ is a slowly varying function and $f(\epsilon)$ is the Fermi-Dirac distribution. The peaks in $J(q)$ probably reflect the contributions of a large number of small-energy denominators at q values corresponding to the separation between approximately parallel sheets of the Fermi surface. These are believed to be vi primary importance in determining $Q$ in the heavy rare earth metals. ${ }^{1}$. Measurements at intermediate temperatures show that these peaks are abruptly flattened at the ferromagnetic transition, and this may be ascribed to the splitting of the different spin bands by the ferromagnetic exchange interaction.

The energy eigenvalues for the conduction electrons in $\mathrm{Tb}$ have been calculated along symmetry lines in the Brillouin zone by the RAPW method, ${ }^{20}$ and part of the band structure is shown in Fig. 12. The energy bands are qualitatively similar to those in the other hcp rare earth metals, except that the $d$ bands are substantially lower in energy than those in, for instance, Dy. ${ }^{21}$ The position of the second lowest doubly degenerate level at $L$ relative to the Fermi level is believed to be of great importance in determining the magnetic ordering in these metals. In most of them it lies above the Fermi level, but in Gd it is below and this is believed to modify the Fermi surface in such a way that $J(\mathbf{q})$ has a maximum at $q=0$, resulting in a stable ferromagnetic structure. This level is also below the Fermi

${ }^{18}$ L. M. Roth, H. J. Zeiger, and T. A. Kaplan, Phys. Rev. 149, 519 (1966).

${ }^{19}$ R. W. Williams, T. L. Loucks, and A. R. Mackintosh, Phys. Rev. Letters 16, 168 (1966).

${ }^{20}$ T. L. Loucks, Phys. Rev. 139, A1333 (1965).

${ }^{21}$ S. C. Keeton and T. L. Loucks, Phys. Rev. (to be published). level in $\mathrm{Tb}$, and this may partially account for the stron tendency towards ferromagnetism, and the anomalous small Q in the spiral phase.

Since the neutron group widths are neither observabl temperature dependent at high $\mathrm{q}$ nor significantly different in the alloy from those in pure Tb, the princ: pal mechanism limiting the magnon lifetime is probabl absorption by the conduction electrons. ${ }^{22}$ This proces involves a spin-flip by the electron, and the absorptio may therefore decrease rapidly at critical wavevector determined by the separation of the Fermi surfaces different spin. For the free-electron model, the width well above the critical $\mathrm{q}$ is given by ${ }^{22}$

$$
\Delta \epsilon(\mathrm{q})=c \epsilon(\mathrm{q}) / q,
$$

where $c$ is a constant. This function, fitted at $q=0.72$ $\AA^{-1}$ is shown in Fig. 7. The rapid fall in the widths at about $q=0.35 \mathrm{~A}^{-1}$ may reflect the exchange splitting o the Fermi surface, and it would clearly be of interest to study this phenomenon as a function of magnetic ordering. The residual broadening at low $\mathrm{q}$ is probably due to Umklapp processes of the type discussed by Luther and Tanaka. ${ }^{23}$

Because of their smaller spin, the Ho moments in the alloy are coupled comparatively weakly to the Tb host moments and so have a natural precession frequency in the band of magnon energies. The resonant scattering of the magnons by the impurities causes a strong perturbation of the dispersion relation in the vicinity of this frequency, and this probably explains the anomaly which is shown in Fig. 6. This resonant magnon mode is discussed in more detail elsewhere."

Finally we discuss the magnon-phonon interaction, which is observed when the unperturbed magnon and transverse phonon dispersion curves cross. If a linear coupling between the excitations is assumed, the Hami

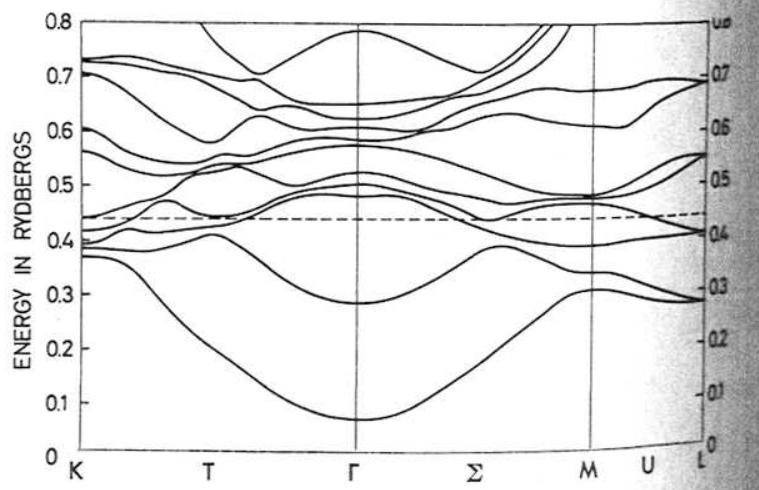

FIG. 12. Conduction-electron energy bands along symmetr directions in $\mathrm{Tb}$. The dashed line is the approximate Fer level.

${ }^{22}$ R. J. Elliott and H. Stern, Inelastic Scattering of Newtines (IEAE, Vienna, 1961)

${ }^{23} \mathrm{~A}$. H. Luther and T. Tanaka (to be published).

${ }_{24}$ A. R. Mackintosh and H. B. Møller, Proceedings of Localines

Excitations Conference, Irvine, California, 1967 (to be published. 


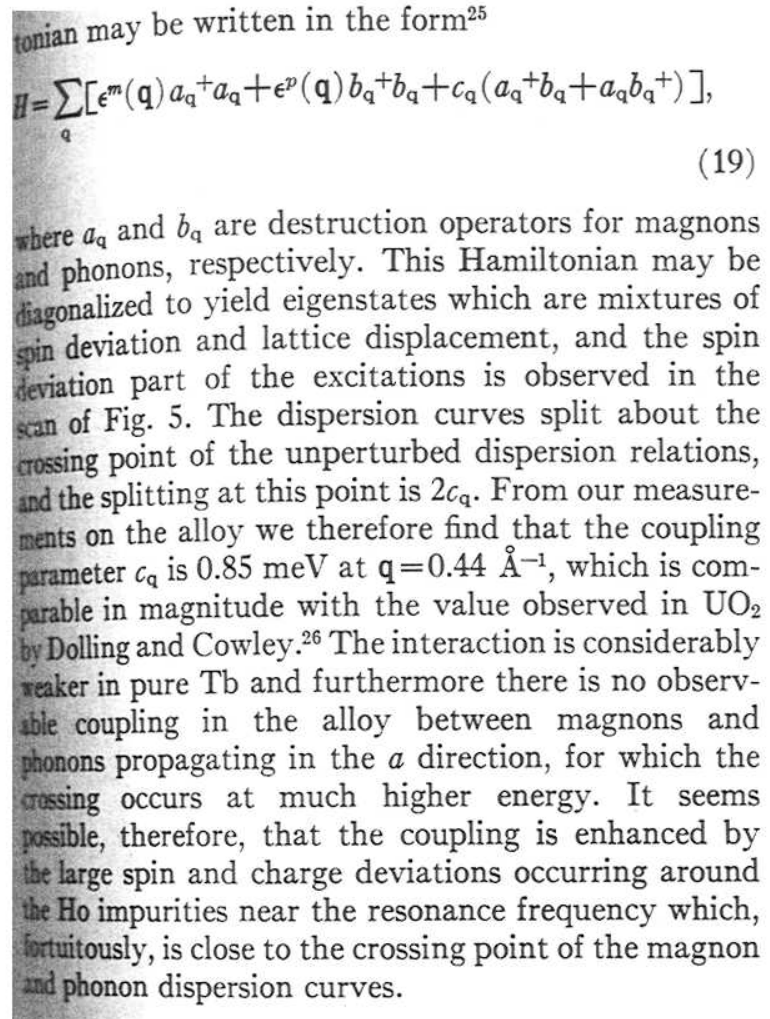

\section{CONCLUSIONS}

From our measurements of the magnon dispersion reations we have been able to obtain a considerable thount of information about the magnetic interactions to the heavy rare earth metals. The indirect exchange cupling between the ions has been studied under rarious experimental conditions, and its form can be reated to the Fermi surface. The temperature dependese reflects the effect of the change in the ordered Donent on the magnon energies and on the conduction sectron energy bands. The exchange splitting of the sergy bands is also manifested in the magnon lifeanes, since the interaction of magnons with conduction electrons apparently drops rather rapidly at a critical q determined by this splitting. The resonant scattering of the magnons from Ho impurities produces a pronounced anomaly in the dispersion relation. A strong coupling occurs between magnons and transverse phonons propagating in the $c$ direction, which may be enhanced by the presence of the resonance.

There are a number of extensions, both experimental and theoretical, which could be made to this work. A more detailed study of the magnon energies and lifetimes as a function of temperature would provide further information on the relation between the conduction electrons and the magnetic interactions. In particular, a study of the magnon energies in the spiral phase should show the effect of the progressive modification of the energy bands by the magnetic ordering, and this should also be reflected in the magnon lifetimes in the ferromagnetic phase, which are critically dependent on the exchange splitting of the bands. From the conduction-electron energy bands, it should be possible to calculate both the exchange coupling between the magnetic ions and the damping of the magnons through absorption by the conduction electrons.

The change in magnon energies with magnetic ordering, and hence with temperature, should allow a more detailed study of the energy of the resonant mode and the strength of the magnon-phonon coupling as a function of q. We are planning soon to extend our measurements down to liquid $\mathrm{He}$ temperatures.

It should also be possible to make detailed measurements on other rare earth metals and alloys in different magnetically ordered phases. Preliminary measurements have already been made on $\mathrm{Er}^{4}$ and $\mathrm{Ho}^{5}{ }^{5}$ We expect that the maxima in $J(\mathbf{q})$ are more pronounced in these metals, since they form periodic magnetic structures over the whole temperature range of ordering. Such experiments, together with theoretical studies of the magnetic interactions, should substantially improve our understanding of magnetism in the rare earth metals.

\section{ACKNOWLEDGMENTS}

We have benefitted greatly from discussions with R. J. Elliott, J. R. Schrieffer, M. F. Collins, W. C. Marshall, M. Blume. T. L. Loucks, G. Johansen, and others. 